\title{
SwarmMesh: A Distributed Data Structure for Cooperative Multi-Robot Applications
}

\author{
Nathalie Majcherczyk Student Member, IEEE and Carlo Pinciroli Member, IEEE \\ Robotics Engineering, Worcester Polytechnic Institute, MA, USA \\ Email: \{nmajcher,cpinciroli\}@wpi.edu
}

\begin{abstract}
We present an approach to the distributed storage of data across a swarm of mobile robots that forms a shared global memory. We assume that external storage infrastructure is absent, and that each robot is capable of devoting a quota of memory and bandwidth to distributed storage. Our approach is motivated by the insight that in many applications data is collected at the periphery of a swarm topology, but the periphery also happens to be the most dangerous location for storing data, especially in exploration missions. Our approach is designed to promote data storage in the locations in the swarm that best suit a specific feature of interest in the data, while accounting for the constantly changing topology due to individual motion. We analyze two possible features of interest: the data type and the data item position in the environment. We assess the performance of our approach in a large set of simulated experiments. The evaluation shows that our approach is capable of storing quantities of data that exceed the memory of individual robots, while maintaining near-perfect data retention in high-load conditions.
\end{abstract}

\section{INTRODUCTION}

Recent work studies with the integration of multi-robot systems with centralized computation platforms, such as databases or cloud computing systems. This approach enables one to aggregate information in a central location and perform efficient map merging, task allocation, and global state estimation - in other words, combining data storage with computational capabilities. This approach is particularly effective in indoor environments, such as warehouses, production chains, and hospitals, in which communication with a central system can be expected to be reliable.

However, many applications are not easily amenable to this approach. Mapping in remote locations, space applications, and disaster recovery are examples in which access to a centralized infrastructure is problematic, heavily limited, or even impossible. In these applications, rather than envisioning a multi-robot system as part of a larger infrastructure, it would be desirable for it to be the infrastructure. These applications also entail the collection of large amounts of data, whose storage might exceed the capacity of any individual robot.

As a step in this direction, we study the realization of a decentralized data structure for storing, managing, and performing computation with shared data. We make three basic assumptions:

- Every robot devotes a quota of memory and bandwidth to storing and routing data. The amount of memory can change across robots;

- The amount of data that the robots must store is larger than the memory capacity of any individual robot;

- The network topology is dynamic due to robot motion.

Given these assumptions, we study how to distribute the data across the swarm. In designing a solution, we realized that in many applications certain features of the data play an important role for mission success. For example, missioncritical data should be stored in well-connected robotsin case of a temporary disconnection this data would be as widely available as possible. Analogously, the physical location of the data might suggest that certain robots are more suitable for storage than others.

The rest of this paper is organized as follows. In Section II we discuss related work. The design of our data structure is presented in Section IV We report the results of our performance evaluation in Section $\mathrm{V}$, and conclude the paper in Section VI.

\section{RELATED WORK}

In peer-to-peer networks, common implementations of data sharing involve Distributed Hash Tables (DHTs). DHTs couple a distributed key partitioning algorithm and a structured overlay network to provide a self-organized data storage service. Information is abstracted in the form of tuples which are (key, value) pairs. The fundamental problem is to decide how to distribute tuples between nodes for storage. The key partitioning algorithm assigns ownership of a set of keys to each node in the network. The overlay network imposes a routing structure that makes for efficient search across the nodes. Comparative surveys [1],[2] highlight the main features of these protocols. The ContentAddressable Network (CAN) protocol partitions the key space by splitting a virtual toroidal space into zones. CAN maps tuples to points owned by nodes in the virtual space using a uniform hash function [3]. In the Chord [4], Pastry [5], and Tapestry [6] protocols, nodes determine NodeIDs according to the structure of the desired overlay network. Tuples are then addressed directly to NodeIDs or partial NodeIDs. These distributed data structures provide selforganizing, scalable and addressable storage. However, node additions and removals are costly as the topology needs to be maintained through reorganization. Furthermore, they can cause local network failures. Because they form relations 
between nodes randomly, unstructured overlay networks such as Gnutella and BitTorrent [7] provide alternatives when the network participant turnover is high. These protocols offer robustness to node removals at the cost of increased degree of centralization or loss of guarantees when locating data.

In the above mentioned protocols, the selection of neighboring nodes in the overlay network lacks physical meaning. This means that neighbors in the network could be far away from each other. Since routing information over longer geographical distances increases energy consumption and latency, there has been an effort to incorporate node location into overlay networks. Three main trends exist within this body of research: (1) Geographic layout, which constructs the overlay network so that neighbors are close in the physical space [8], [9], [10], [11]; (2) Proximity routing, which considers node proximity while routing in the existing overlay network [12]; (3) Proximity neighbor selection, which weighs in proximity between neighbors when constructing the overlay network [13]. These methods add a notion of node locality. However, the network topology only changes to accommodate node additions and removals but not motion. Therefore, they fail to capture the inherent dynamicity of robotic systems.

There is a vast body of research in Mobile Ad-Hoc Networks (MANETs) that seeks to address communication between mobile interconnected devices. Some sensor networks have motion and fall in that category. One trend in those systems has been to use naming and data-centric routing and storage. This means that a name is associated to given data and that name determines to which node the data is addressed [14]. Similarly to swarm systems, the main features of sensor networks are that they are limited in energy, memory and computational power. Sensor networks perform distributed data processing and storage. However, the goal is to eventually offload the processed data to a base station. Furthermore, sensors typically do not act on the environment or perform cooperative and autonomous decision-making. Vehicular Ad Hoc Networks (VANETs) are systems of interconnected cars and road stations. Different types of routing protocols have been studied within that field, they can be divided into: proactive routing, reactive routing and position-based routing which depends on beaconing and forwarding [15], [16], [17]. These systems share some similarities with swarms but differ in that they have specific topologies and mobility patterns. Typically, cars in the back make decisions based on cars up front. The lanes and roads are narrow so the number of direct neighbors is small.

Several papers compare and assess the use of existing databases in multi-robot applications [18], [19] and [20]. These comparisons reveal that most existing databases rely on a central server. An exception is the work of Sun et al. [21], who adapted Distributed Heterogeneous Hash Tables and position-based routing to propose a solution for task allocation in a warehouse setting. In the context of swarm robotics, Pinciroli et al. proposed a distributed tuple space called virtual stigmergy [22] that copes with frequent topology changes. In this approach, each robot maintains a local time-stamped copy of the data which is only accessed upon read and write operations. This mechanism works well with node mobility and limited bandwidth but it leads to full data duplication. This means that the collective memory of the system is severaly under-utilized. The SOUL file sharing protocol [23] builds on virtual stigmergy and unstructured overlay networks to enable sharing of larger-size data in the form of (key, blob) pairs. SOUL involves locally storing blob meta-data on each node and splitting blobs into datagrams across different nodes. This decomposition uses a bidding mechanisms that minimize the reconstruction cost at so-called processor nodes. This method addresses the problem of managing data files with a focus on how to split, distribute and recombine them. Memory usage is improved but meta-data is still fully duplicated across nodes for each of the files. Various update and bidding processes increase latency in the network.

In this paper, we take inspiration from existing methods and propose a novel approach to distributed data sharing. Our design embraces the decentralized nature of robot swarms and the constant change and volatility in the network topology that results from robot motion. We organize our data flow based on instantaneous local properties at each node so as to get a memory efficient, consensus-free approach with a low communication overhead.

\section{Problem StATEMENT}

In this section, we describe the fundamental assumptions imposed both on the multi-robot system and on the nature of the events to record in the physical environment. We proceed by describing challenges of the distributed storage problem in this context.

\section{A. Ad-hoc Robotic Network}

We consider an autonomous and decentralized system of $N$ robots which act as both the infrastructure for storing information and sole users of this information. We define the system across the following features:

a) Communication Modalities: We assume that the robots have the ability to exchange data within a communication range $C$. This implies the existence of an adhoc network with each robot acting as a node. We further assume that robot communication is limited to gossiping, i.e., broadcasting messages to all neighbors within $C$. Because we also desire to route some messages from one robot to another using the point-to-point communication modality, we assume that the robots have a constant unique identifier $i \in[1, N]$ and a variable node identifier $\delta_{i}$ made known to their neighbors. The knowledge of $i$ singles out a specific robot, while $\delta_{i}$ enables the selection of a suitable storage node for a specific tuple.

b) Finite Resources: We impose a realistic finite bandwidth on outgoing messages. We also limit the memory capacity $M_{i}$ of each robot allotted for the self-organizing data management process. Variable $m_{i}(t)$ records the amount of memory used by robot $i$ at a given time. 
c) Dynamic Topology: The robots are moving according to a logic defined by the developer. Robot motion follows linear dynamics and has a limited speed. The number of neighbors $n g b r s_{i}(t)$ of a robot changes over time.

\section{B. Inputs}

We consider inputs to the data structure to stem from events which have a position $\mathbf{x} \in \mathbb{R}^{d}$ and happen at a time $t \in \mathbb{N}$. Such events can be, e.g., records of a physical phenomenon sampled at a particular time and place, records of an internal robot state or records of swarm-wide state. To implement a data structure in which robots can retrieve and update tuples encoding some events, each specific tuple needs a unique identifier $\tau$ meaningful to all network nodes. In particular, for updates, tuples need to have a notion of version. For convenience, we achieve versioning by timestamping tuples with a global time. Distributed synchronization algorithms such as vector clocks can be used to implement this aspect.

\section{Methodology}

\section{A. Overall Architecture}

We describe our design following the structure depicted in Figure 1. SwarmMesh provides algorithms across three levels of abstraction.

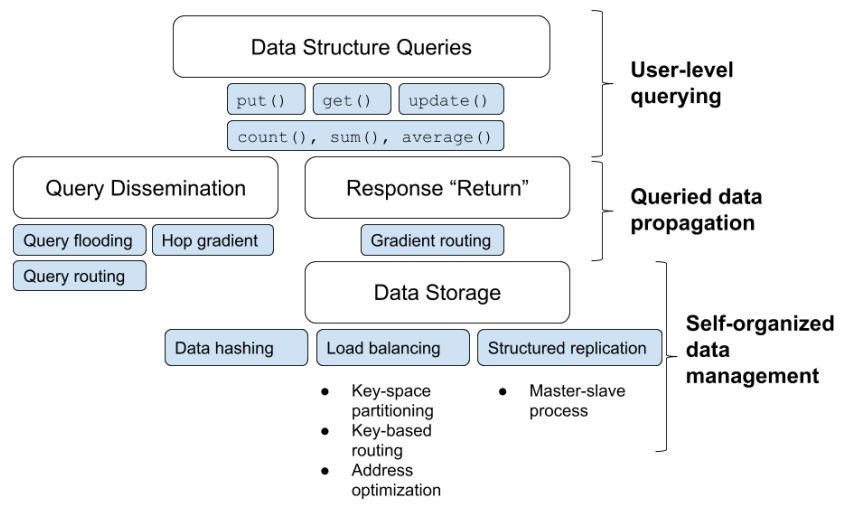

Fig. 1: Overall architecture.

1) User-level querying: As stated in Section I, robots are at the same time the networking infrastructure and users of the data stored by the network. As a user, a robot can execute different querying commands on the data structure. These operations are meant to enable modifying and retrieving information stored globally as required by the robot behavior. This behavior is defined by the developer and independent from SwarmMesh.

2) Queried data propagation: Another layer of SwarmMesh handles the dissemination of user read and write queries throughout the data structure. Read queries are flooded across the network. This type of query requires replies from certain nodes to be routed back to the robot emitting the query. We route write operations to a suitable node for storage in a point-to-point fashion.
3) Self-organized data management: The bottom layer determines how the tuples get distributed across nodes. It also ensures a certain degree of robustness by creating inactive replicas in other nodes in a controlled way. The main design intuitions driving the data distribution are that: (1) some events/tuples are more important than others (hierarchy in data hashing); (2) some nodes are better suited to hold more valuable tuples than others (hierarchy in key-space partitioning); (3) the hierarchy of nodes changes very often and should be updated based on local information only.

\section{B. User-level Querying}

A robot user can perform the following operations:

- $\operatorname{put}(\mathrm{k}, \mathrm{V})$ : writes a tuple into the data structure. It performs an erase $(\mathrm{k})$ to remove any potential outdated version of the tuple and a store $(\mathrm{k}, \mathrm{v})$ of the new tuple.

- store $(\mathrm{k}, \mathrm{v})$ : assigns a tuple to a particular node in the data structure.

- erase $(\mathrm{k})$ : removes a tuple from the data structure.

- get $(\mathrm{k}, \Delta)$ : returns all the values corresponding to keys $\in[k-\Delta ; k+\Delta]$ (see Figure 2).

- get $(x, y, r)$ : returns all the values for tuples located within a radius $r$ of the point $(x, y)$ expressed in a global reference frame. To use this feature, we need the added assumption of a global reference frame and the ability to locate events in this reference frame (see Figure 2).

Robots can also perform in-network computation:

- count $(k, \Delta)$ or count $(x, y, r)$ : returns the number of tuples with keys $\in[k-\Delta ; k+\Delta]$ or located within a radius $r$ of the point $(x, y)$.

- $\operatorname{sum}(k, \Delta)$ and $\operatorname{sum}(x, y, r):$ returns the sum of values corresponding to keys $\in[k-\Delta ; k+\Delta]$ or located within a radius $r$ of the point $(x, y)$.

- average $(\mathrm{k}, \Delta)$ or average $(\mathrm{x}, \mathrm{y}, \mathrm{r})$ : returns the result of the corresponding count () and sum() operations as a pair.

- $\min (x, y, r)$ or $\min (k, \Delta)$ : returns the minimum value in the associated spatial range or key range.

- $\max (x, y, r)$ or $\max (k, \Delta):$ returns the maximum value in the associated spatial range or key range.

As explained in Section III-B a write operation should be the result of some local information processing performed by robots in the vicinity of an event. Existing methods in sensornets can be applied to locally synthesize low-level sensor readings into a result describing a higher level event such as source detection [24]. In order to trigger a single data structure write in the vicinity of the event, we locally elect a leader to perform a put $(\mathrm{k}, \mathrm{v})$ operation. The election logic can be redefined by the developer, although the specifics of this aspect are beyond the scope of this paper.

The return values of the count(), sum() and average () operations percolate across nodes. The user robot which emitted the initial query must combine the

\footnotetext{
${ }^{1}$ The key argument $\mathrm{k}$ has an uniquely identifying part $\tau$ and a contentdependent part (or hash) $\rho_{\tau}$.
} 


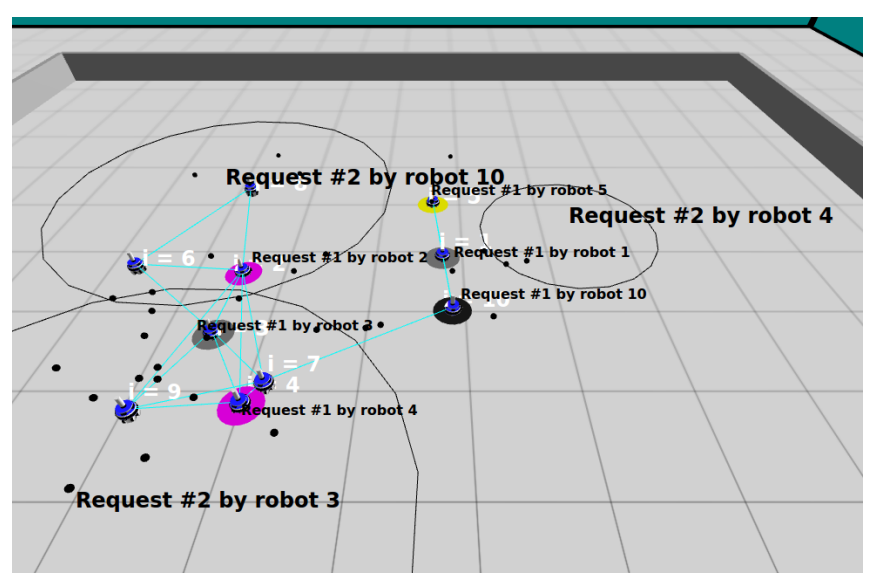

Fig. 2: Black dots show locations of events previously written into the data structure. Queries of the type get $(x, y, r)$ are drawn with black circles representing the area covered. Queries of type get ( $k, \Delta=0$ ) are represented by colored disks under the query source robot; the color maps to a specific key.

intermediate return values into the final result. Monotonic (i.e., commutative and associative) operations such as min () and $\max ()$ do not require combining intermediate results.

The performance of spatial queries, i.e., operations with arguments $(x, y, r)$, and that of queries by key, i.e., operations with arguments $(k, \Delta)$, depends heavily on the way we distribute the data in the network. Our approach is meant to be modular and we present two possible data hashing functions in Section IV-D.1

\section{Queried Data Propagation}

1) Read-operation flooding: Queries that aim to retrieve data from the data structure are flooded to all nodes. Each robot emitting a read query computes the query's unique identifier by concatenating the value of its query counter and its robot unique identifier.

2) Hop gradient: While flooding the network with a read query, we opportunistically create a gradient to the source of the query. Upon reception, every robot increments a hop counter included in the query message and broadcasts it further along. For each received request, the robot stores the query unique identifier and hop count in a circular buffer.

3) Reply gradient routing: The hop gradient gives us a convenient way to route replies back to the source node by forwarding replies from nodes with a higher or equal hop count. For this, we rely on the assumption that the motion of the robots preserve a gradient path to the source for long enough, which is a realistic assumption in most settings when comparing motion speed and information propagation speed.

4) Write-operation routing: When a robot writes the result of some local information processing to the data structure, the tuple may be routed to a different robot for storage based on its key. This algorithm is described in Section IV-D.3.

\section{Self-organizing Data Management}

1) Data Hashing: When writing a tuple using put $(\mathrm{k}, \mathrm{v})$, the robot must compute the key $\mathrm{k}$. In our protocol, a key should be in the format $k_{\tau}=\left(\tau, \rho_{\tau}\right)$ where $\tau$ is a tuple unique identifier and $\rho_{\tau}$ is a value that maps to one or multiple nodes which can store the tuple.

The robot assigns $\tau$ by concatenating its robot unique identifier and the count of tuples it has written into the data structure. Each field has a set number of digits so that every $\tau$ is unique. As stated previously, our design considers that events vary in importance and we use this property to distribute them across nodes.

Read queries described in Section IV-B can either use $\mathrm{k}=\rho_{\tau}$ or $\mathrm{k}=\left(\tau, \rho_{\tau}\right)$ for tuple addressing. Queries for $\rho_{\tau}$ can yield multiple tuples while queries in $\left(\tau, \rho_{\tau}\right)$ relate to a specific tuple.

The robot computes $\rho_{\tau}$ using a function mapping a characteristic of the event to its relative importance. We select the function such that the higher $\rho_{\tau}$, the more valuable the piece of information. We propose two hashing functions:

- Category-based: We consider that a robot can register different types of events. For example, it can mean that the robot has several different on-board sensors and determines the event type by the triggered sensor. We use a ranking function $R_{T}\left(s_{\tau}\right)$ that assigns higher values to event types that we consider most important: $h_{C}:$ type $_{\tau} \mapsto \rho_{\tau}=R_{T}\left(\right.$ type $\left._{\tau}\right)$

- Spatial: We decide that in a global reference, tuples further away from the origin are the most desirable because they are difficult to discover by robots. This idea can be generalized to specific areas in any reference frame: $h_{S P}:\left(x_{\tau}, y_{\tau}\right) \mapsto \rho_{\tau}=\sqrt{x_{\tau}^{2}+y_{\tau}^{2}}$.

2) Key Partitioning: Similarly to other distributed data structures such as DHTs, nodes partition the key space to decide which one of them needs to hold tuples corresponding to specific keys.

As stated in Section IV-A, we use the idea that some nodes are superior than others. Our intuition is that a robot with more neighbors $n g b r s_{i}$ is less likely to get disconnected from the swarm and is better positioned to dispatch tuples upon query. A second insight is that the more free data memory a robot has, the less likely it is to overflow its memory and discard information. We also desire to have instantaneous self-organized partitioning completely based on local information. This leads us to make nodes assign themselves a node identifier $\delta_{i}$ as follows:

$$
\delta_{i}(t)= \begin{cases}m_{i}(t) \cdot n g b r s_{i}(t) & \text { if } n g b r s_{i}(t)>0 \\ 1 & \text { otherwise }\end{cases}
$$

A node with node identifier $\delta_{i}$ can hold a tuple with key $\left(\tau, \rho_{\tau}\right)$ if $\delta_{i}(t)>\rho_{\tau}$. We refer to this condition as $(\mathrm{H})$ in the rest of this text. In order to store tuples in the data structure, we should match the frequency distributions of data hashes and node identifiers, i.e., there should be nodes with unique identifiers at least high enough to hold the hashed tuples. This has implication on the design of the hashing functions. 
They should map to values smaller than $\max _{i}\left(M_{i}\right) \cdot(N-1)$ and spread the data across likely node identifiers.

3) Key-based Routing: If a robot holds one or more tuples not satisfying $(\mathrm{H})$, it places them in a routing queue. It then tries to send them starting with the highest $\rho_{\tau}$ to a robot with a high enough node identifier. If there are candidates satisfying $(\mathrm{H})$ to receive the tuple, the sender picks one at random. If none of the neighbors satisfy the condition, the robot sends it to the neighbor of highest $\delta_{i}$. We impose a limit on the memory capacity $M_{i}$ and divide it into routing and storage capacities. In case of overloads on $M_{i}$, the robot discards the least important tuple, i.e., with lowest $\rho_{\tau}$.

4) Address Optimization: When a robot has an empty routing queue and it stores a tuple with $\rho_{\tau}$ closer to the node identifier of a candidate neighbor, we let the robot evict the tuple to the corresponding neighbor. This is an optimization to ensure efficient access to a tuple by key. We further noticed that requiring at least a half full storage memory helps balancing the load between nodes.

5) Structured Replication: To ensure robustness to node failures, we make copies in neighboring nodes using a master-slave approach. The master is the robot holding the original tuple. It picks a slave to hold an inactive copy of the tuple. Robots do not return inactive tuple copies upon queries; this ensures consistency. Master and slave exchange a heartbeat signal. If the master fails to receive the heartbeat signal within a time-out duration, it picks another slave. The master can also send a kill signal to cancel the inactive copy. The master cancels the copy if the slave gets outside of a safe radius of communication $(\ll C)$ or if it decides to route the active tuple to another robot. If a slave fails to receive the heartbeat within the time-out duration from the master, it activates the tuple copy.

\section{Evaluation}

\section{A. Metrics and Parameters}

We evaluated different aspects of our approach such as scalability, memory-related performance, and routing protocol efficiency.

To study scalability, we performed our simulated experiments with 10, 50 and 100 robots uniformly distributed inside an arena sized to impose densities of 0.6 and 1 robot $/ \mathrm{m}^{2}$. We set the robot communication range to $2 \mathrm{~m}$. These densities imply that the ad-hoc network stays often connected even with diffuse robot motion. This enables us to study a system facing intermittent disconnections.

In hash tables, the load factor is the number of data items over the number of memory slots (buckets). This parameter indicates the load of the data structure and is typically used to decide when to partition of the memory into an increased number of buckets. For our distributed and self-organized approach, we define the load factor as $l_{f}=$ number of events $/(N \cdot S)$, where $S$ is the storage capacity. The memory capacity $M$ includes both storage and routing capacities.

To understand the performance of the key-based routing algorithm, we track the number of hops and time steps for
TABLE I: Simulation parameters

\begin{tabular}{|lc|}
\hline Parameter & Value \\
\hline Number of Robots $N$ & $\{10,50\}$ robots \\
Communication range $C$ & $2 \mathrm{~m}$ \\
Memory capacity $M_{i}$ & 20 tuples $\forall i$ \\
Storage capacity $S$ & 10 tuples \\
Routing capacity $R$ & 10 tuples \\
Time step & $0.1 \mathrm{~s}$ \\
Bandwidth & $5.7 \mathrm{kB} / \mathrm{s}$ \\
Robot density & $\{0.6,1\} \mathrm{robot} / \mathrm{m}^{2}$ \\
Robot speed & $\{0,5\} \mathrm{cm} / \mathrm{s}$ \\
Load factor & $0.6,0.7,0.8,0.9,1\}$ \\
Event sensing range & $1 \mathrm{~m}$ \\
Event types & 12 \\
Events generation rate & 5 events/s \\
Query generation rate & 1 query/s \\
\hline
\end{tabular}

a tuple to be routed to a suitable node for storage and upon query. We implemented messaging with a queue and we imposed a limit on the bandwidth for outgoing messages. We only send one tuple at a time.

To study availability, we considered the fraction of tuples received over the expected tuples for a get () query. We checked for consistency by confirming that active copies of tuples were all unique.

\section{B. Simulated Experiments}

We tested our system according to the described metrics in the ARGoS multi-robot simulator [25]. We ran simulations with and without robot motion. We picked a simple diffusion motion with a maximum forward speed of $5 \mathrm{~cm} / \mathrm{s}$. For the purpose of testing all available features, robots are equipped with a range and bearing sensor $(C=2 \mathrm{~m})$, a GPS and a sensor detecting colored spheres. We disabled line-of-sight obstructions. To materialize events, we put colored spheres in the environment with each color representing a category of event. Events were generated in time according to a Poisson distribution and placed in space according to a uniform distribution.

1) Memory-Related Performance: In our simulations, we allocate limited memory and bandwidth to the data sharing process. Upon receiving tuples that it can store, a robot progressively fills its storage memory. The cap on bandwidth combined with the decision to route one tuple per time step results in some tuples being temporarily placed in a routing memory. The goal is to keep the storage memory under a value $S$ and the routing memory under a value $R$. However, we allow either memory to temporarily cross that threshold provided that the combined memory usage stays under the memory capacity $M_{i}$. Any memory overflow leads to robots discarding tuples of lowest rank. To assess the ability to retain large amounts of information in the data structure, we generate different numbers of inputs corresponding to load factors between 0.6 and 1.0. We repeated simulations with and without robot motion and using either the $h_{C}$ or $h_{S P}$ hash function. Tables II and III show that the fraction of retained tuples is almost always equal to 1 , even with high load factors. This indicates that the collective memory is properly utilized with robots sharing the data load. In comparison, an approach that uses full duplication and the 


\begin{tabular}{llllllll} 
load factor & & & $\mathbf{. 6}$ & $\mathbf{. 7}$ & $\mathbf{. 8}$ & $\mathbf{. 9}$ & $\mathbf{1}$ \\
\hline \hline \multirow{4}{*}{ category } & \multirow{2}{*}{ static } & $\min$ & 1 & 1 & 1 & .996 & .95 \\
hashing & topology & $\operatorname{mean}$ & 1 & 1 & 1 & .999 & .983 \\
& & $\max$ & 1 & 1 & 1 & 1 & 1 \\
\cline { 2 - 8 } & \multirow{2}{*}{ dynamic } & $\min$ & 1 & 1 & 1 & .996 & .986 \\
& topology & $\operatorname{mean}$ & 1 & 1 & 1 & .999 & .992 \\
& & $\max$ & 1 & 1 & 1 & 1 & 1 \\
\hline \hline \multirow{4}{*}{ spatial } & \multirow{2}{*}{ static } & $\min$ & .993 & .932 & .973 & .909 & .758 \\
hashing & topology & $\operatorname{mean}$ & .999 & .99 & .987 & .948 & .899 \\
\cline { 2 - 8 } & & max & 1 & 1 & 1 & .98 & .954 \\
\cline { 2 - 9 } & \multirow{2}{*}{ dynamic } & $\min$ & .997 & .994 & .985 & .984 & .94 \\
& topology & $\operatorname{mean}$ & .999 & .999 & .996 & .993 & .985 \\
& & $\max$ & 1 & 1 & 1 & 1 & .998
\end{tabular}

TABLE II: Tuple retention for $N=50$ across load factors.

\begin{tabular}{lllllll} 
load factor & & & $\mathbf{. 6}$ & $\mathbf{. 7}$ & $\mathbf{. 8}$ & $\mathbf{. 9}$ \\
\hline \hline \multirow{4}{*}{ category } & static & $\min$ & 1 & 1 & 1 & .997 \\
hashing & topology & $\operatorname{mean}$ & 1 & 1 & 1 & .999 \\
& & $\max$ & 1 & 1 & 1 & 1 \\
\cline { 2 - 7 } & \multirow{2}{*}{ dynamic } & $\min$ & 1 & 1 & .991 & .977 \\
& topology & $\operatorname{mean}$ & 1 & 1 & .999 & .996 \\
& & $\max$ & 1 & 1 & 1 & 1 \\
\hline \hline \multirow{4}{*}{ spatial } & \multirow{2}{*}{ static } & min & .955 & .912 & .841 & .72 \\
hashing & topology & $\operatorname{mean}$ & .982 & .97 & .927 & .866 \\
& & $\max$ & 1 & .999 & .989 & .977 \\
\cline { 2 - 7 } & \multirow{2}{*}{ dynamic } & min & .995 & .993 & .994 & .972 \\
& topology & mean & .999 & .999 & .998 & .997 \\
& & max & 1 & 1 & 1 & 1
\end{tabular}

TABLE III: Tuple retention for $N=100$ across load factors.

same individual memory constraints would retain $N$ times less tuples (excluding the routing memory).

In order to evaluate key partitioning, we show histograms of node identifiers and data hashes across all simulations with 100 robots in Figure 3 In Figure 3a, we use the categorybased hash function $h_{C}$ with a mapping of 12 types of events to values in $\{1,11,21, \ldots, 121\}$. We generated the types of events uniformly in the simulations which naturally leads to the white dashed bar graphs in Figure 3a. Node identifiers are the product of the current node degree in the communication graph and the node's remaining storage memory. Therefore, the node identifier distribution depends on a combination of communication graph topology and load allocation. Both situations represented for the category-based have the node identifier distribution to the right of the data hashes. In the upper graph, robots are static and their spatial coordinates are sampled in uniform distributions. In the bottom graph, robots diffuse in an arena sized to impose certain robot densities (see Table II). In Figure 3b, we use the spatial hash function $h_{S P}$ and we show a situation where events are uniformly generated up to $8 \mathrm{~m}$ from the origin of the global reference frame. The function $h_{S P}$ maps the distance in $\mathrm{cm}$ to $\rho_{\tau}$ which yields the Gaussian distribution represented by the white bar graphs. In both the static and moving case, the node identifier distribution is to the left of the data keys distribution. This means that, given the key partitioning condition $(\mathrm{H})$, suitable nodes for storing tuples are scarce or non-existent. As evidenced by Table [III, we were still able to retain tuples with high load factors even in this situation. The reason is that robots shift the load from their storage memory to their routing memory. This is apparent in Figure $3 \mathrm{c}$ in which the number of tuples in storage memory normalized by the total number of tuples shows the difference between the use of $h_{C}$ and $h_{S P}$. With the latter, most tuples remain in routing and bounce between robots with more free memory. This is not a desirable solution as it increases the communication overhead. However, it demonstrates a certain tolerance and seamless adaptation to inappropriate node partitioning. In practice, with a guess of the environment scale and typical distances, $h_{S P}$ can be scaled so as to provide mapping to a range matching the node identifiers.

2) Routing Performance: In our approach, routing mechanisms depend on the type of query. A write operation put () triggers a flooded erase() operation and a store() propagated through key-based routing (see Section IV-D.3 . The timing for storing a tuple depends on how difficult it is to reach a suitable node given the tuple key. Figure 4 shows the median routing time with 10 and 100 robots for the store () operation. The time tends to increase with the key indicating that lower range keys find a match faster.

Read operations of any type generate a message flooded to all robots. Replies come back through gradient routing (see Section IV-C.3. Figure 5 reports the median duration between a robot emitting a spatial get () query and receiving the last reply to the query. This duration tends to increase with the network size and with the radius of the query.

3) Message Load: The outgoing bandwidth was set to 570 bytes per time step for each robot, with a time step covering $100 \mathrm{~ms}$. However, this allowance was rarely needed. Figure 6 shows the median bandwidth usage across simulations over time, which remains well below the limit we imposed.

\section{CONCLUSIONS}

In this paper we present SwarmMesh, a distributed data structure for low-memory, low-bandwidth, highly mobile multi-robot systems. The main insight in the design of SwarmMesh is that the features that characterize the data items play an important role in deciding where to store the items. Our design is modular, in that the logic that governs data distribution can be chosen by the user. In this paper we focus on two methods of distributing storage responsibility, one based on the category of the data items (for applications in which certain data types are more important than others), and another based on the position of each data item. The results of our evaluation show that SwarmMesh displays near-perfect levels of data retention even for extremely high load factors, adaptively switching from static storage in the robot memory when load factors are low, to dynamic storage through frequent data exchange when load factors are severely high.

Future work involves applying our work to a variety of scenarios, including task allocation in dynamic environments and collaborative mapping. For the latter scenario, we will also investigate how to incorporate the size of the data items as a factor in the data redistribution logic. Finally, our approach lends itself to privacy and security considerations, whereby the decision on where to store certain data depends on the reputation of the robots. 

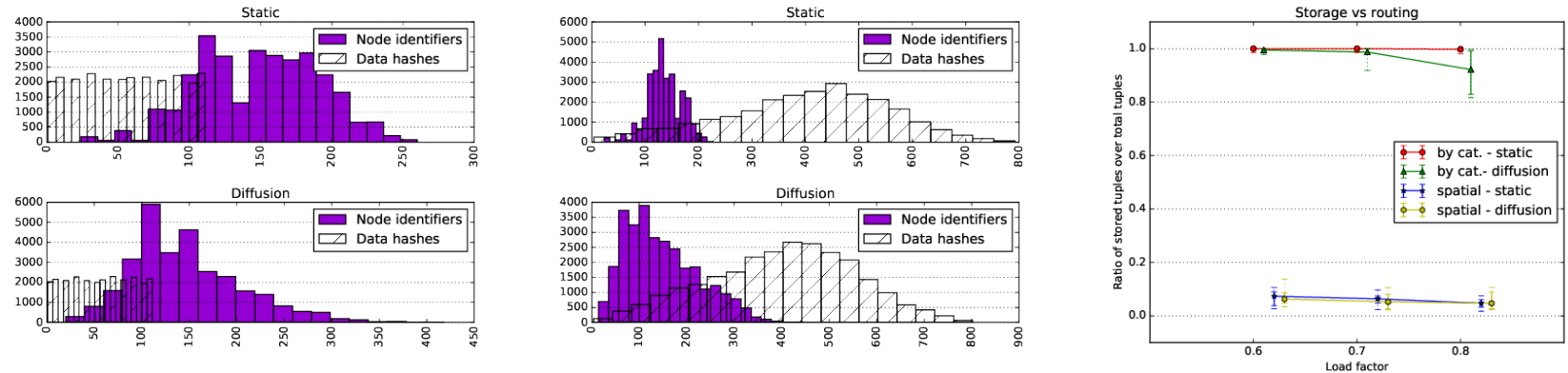

(a) NodeID distribution in category-based (b) NodeID distribution in spatial hashing. hashing.

(c) Ratio of stored to routed tuples.

Fig. 3: Performance of key partitioning with $N=100$ robots.

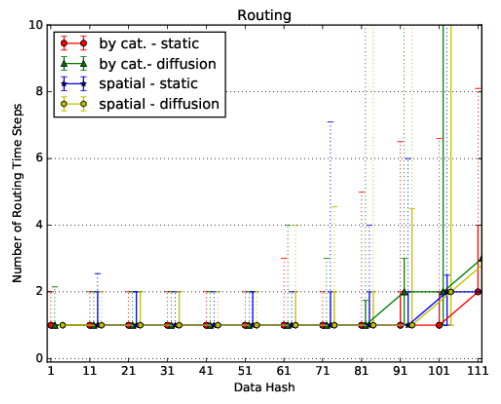

(a) $\mathrm{N}=10$

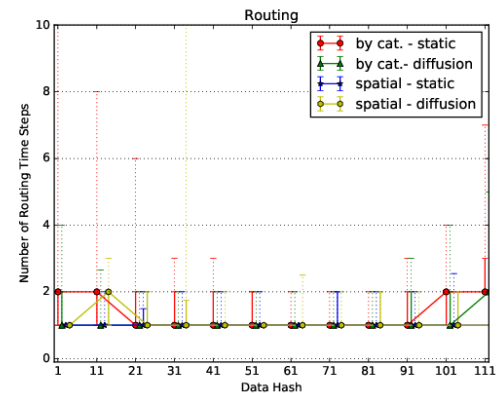

(b) $N=100$

Fig. 4: Number of time steps $(100 \mathrm{~ms}$ each) for the completion of store $(\mathrm{k}, \mathrm{v})$ operations.

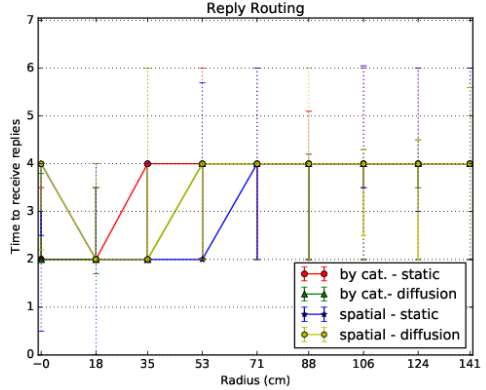

(a) $N=10$

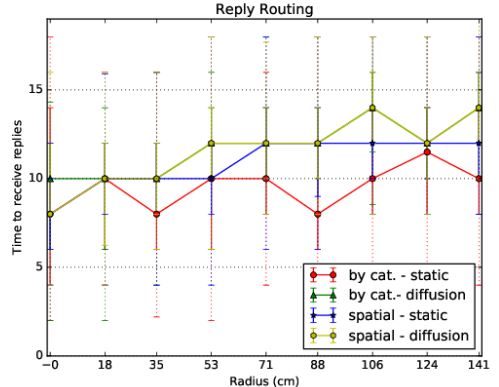

(b) $N=100$

Fig. 5: Number of time steps $(100 \mathrm{~ms}$ each) for the completion of get $(x, y, r)$ queries.

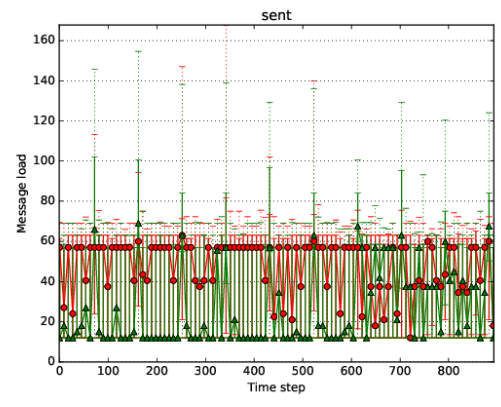

(a) $N=10$

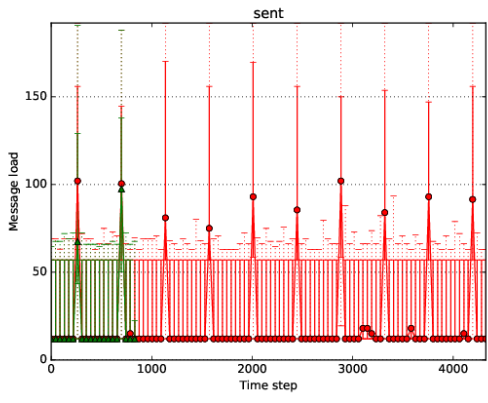

(b) $N=50$

Fig. 6: Bandwidth usage in bytes over time. Time is measured in time steps $(100 \mathrm{~ms})$. 


\section{ACKNOWLEDGMENTS}

This work was funded by a grant from mRobot Technology Co, Shanghai, China.

\section{REFERENCES}

[1] G. Urdaneta, G. Pierre, and M. V. Steen, "A survey of DHT security techniques," ACM Computing Surveys, vol. 43, no. 2, pp. 1-49, 2011. [Online]. Available: http://portal.acm.org/citation.cfm?doid=1883612. 1883615

[2] E. K. Lua, J. Crowcroft, M. Pias, R. Sharma, and S. Lim, "A Survey and Comparison of Peer-to-Peer Overlay Network Schemes," no. March, 2004. [Online]. Available: http://www.sciencedaily.com/ releases/2009/01/090121122836.htm

[3] S. Ratnasamy, P. Francis, M. Handley, R. Karp, and S. Shenker, A scalable content-addressable network. ACM, 2001, vol. 31, no. 4.

[4] I. Stoica, R. Morris, D. Karger, M. F. Kaashoek, and H. Balakrishnan, "Chord: A scalable peer-to-peer lookup service for internet applications," ACM SIGCOMM Computer Communication Review, vol. 31, no. 4, pp. 149-160, 2001.

[5] A. Rowstron and P. Druschel, "Pastry: Scalable, decentralized object location, and routing for large-scale peer-to-peer systems," in IFIP/ACM International Conference on Distributed Systems Platforms and Open Distributed Processing. Springer, 2001, pp. 329-350.

[6] B. Y. Zhao, L. Huang, J. Stribling, S. C. Rhea, A. D. Joseph, and J. D. Kubiatowicz, "Tapestry: A resilient global-scale overlay for service deployment," IEEE Journal on selected areas in communications, vol. 22, no. 1, pp. 41-53, 2004.

[7] B. Cohen, "Incentives build robustness in bittorrent," in Workshop on Economics of Peer-to-Peer systems, vol. 6, 2003, pp. 68-72.

[8] J. P. Ahulló, P. G. López, M. S. Artigas, and A. F. Gómez Skarmeta, "Supporting geographical queries onto DHTs," Proceedings - Conference on Local Computer Networks, LCN, pp. 435-442, 2008.

[9] W. Wu, Y. Chen, X. Zhang, X. Shi, L. Cong, B. Deng, and X. Li, "LDHT : Locality-aware Distributed Hash Tables *," no. 2007.

[10] A. Pethalakshmi and C. Jeyabharathi, "Geo-Chord: Geographical Location based Chord Protocol in Grid Computing," Tech. Rep. 3, 2014. [Online]. Available: https://pdfs.semanticscholar. org/d4e4/0a9bd5845a17030d21228e1be0b9d2065f9c.pdf

[11] S. Matsuura, K. Fujikawa, and H. Sunahara, "Mill: A Geographical Location Oriented Overlay Network Managing Data of Ubiquitous Sensors," 2007. [Online]. Available: http://library.naist.jp/dspace/bitstream/handle/10061/4970/

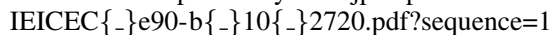

[12] F. Araujo, L. Rodrigues, J. Kaiser, Changling Liu, and C. Mitidieri, "CHR: A Distributed Hash Table for Wireless Ad Hoc Networks," in 25th IEEE International Conference on Distributed Computing Systems Workshops. IEEE, pp. 407-413. [Online]. Available: http://ieeexplore.ieee.org/document/1437204/

[13] M. Castro, P. Druschel, Y. C. Hu, and A. Rowstron, "Exploiting network proximity in distributed hash tables," in International Workshop on Future Directions in Distributed Computing (FuDiCo), 2002, pp. 52-55.

[14] S. Ratnasamy, B. Karp, S. Shenker, D. Estrin, R. Govindan, Y. Li, and Y. U. Fang, "Data-Centric Storage in Sensornets with GHT, a Geographic Hash Table," Tech. Rep., 2003. [Online]. Available: https://link.springer.com/content/pdf/10.1023/A:1024591915518.pdf

[15] S. Zeadally, R. Hunt, Y.-S. Chen, A. Irwin, and A. Hassan, "Vehicular ad hoc networks (vanets): status, results, and challenges," Telecommunication Systems, vol. 50, no. 4, pp. 217-241, 2012.

[16] S. Yousefi, M. S. Mousavi, and M. Fathy, "Vehicular ad hoc networks (vanets): Challenges and perspectives," in 2006 6th International Conference on ITS Telecommunications, June 2006, pp. 761-766.

[17] V. D. Viswacheda, A. Chekima, F. Wong, and J. A. Dargham, "A study on vehicular ad hoc networks," in 2015 3rd International Conference on Artificial Intelligence, Modelling and Simulation (AIMS), Dec 2015, pp. $422-426$.

[18] R. Ravichandran, E. Prassler, N. Huebel, and S. Blumenthal, "A workbench for quantitative comparison of databases in multi-robot applications," in 2018 IEEE/RSJ International Conference on Intelligent Robots and Systems (IROS). IEEE, 2018, pp. 3744-3750.

[19] D. Fourie, S. Claassens, S. Pillai, R. Mata, and J. Leonard, "Slamindb: Centralized graph databases for mobile robotics," in Robotics and Automation (ICRA), 2017 IEEE International Conference on. IEEE, 2017, pp. 6331-6337.
[20] A. J. Fiannaca and J. Huang, "Benchmarking of relational and nosq databases to determine constraints for querying robot execution logs," Computer Science \& Engineering, University of Washington, USA, pp. 1-8, 2015.

[21] D. Sun, A. Kleiner, and C. Schindelhauer, "Decentralized Hash Tables For Mobile Robot Teams Solving Intra-Logistics Tasks," in Proc. of 9th Int. Conf. on Autonomous Agents and Multiagent Systems. IFAAMAS, 2010, p. 10.

[22] C. Pinciroli, A. Lee-Brown, and G. Beltrame, "A tuple space for data sharing in robot swarms," in Proceedings of the 9th EAI International Conference on Bio-inspired Information and Communications Technologies (formerly BIONETICS). ICST (Institute for Computer Sciences, Social-Informatics and , 2016, pp. 287-294.

[23] V. S. Varadharajan, D. St-Onge, B. Adams, and G. Beltrame, "Soul: data sharing for robot swarms," Autonomous Robots, pp. 1-18, 2019.

[24] K. Yao, R. E. Hudson, C. W. Reed, D. Chen, and F. Lorenzelli, "Blind beamforming on a randomly distributed sensor array system," IEEE Journal on Selected Areas in Communications, vol. 16, no. 8, pp. 1555-1567, 1998.

[25] C. Pinciroli, V. Trianni, R. O’Grady, G. Pini, A. Brutschy, M. Brambilla, N. Mathews, E. Ferrante, G. Di Caro, F. Ducatelle, M. Birattari, L. M. Gambardella, and M. Dorigo, "ARGoS: a modular, parallel, multi-engine simulator for multi-robot systems," Swarm Intelligence, vol. 6, no. 4, pp. 271-295, 2012. 